\title{
Esthetic and Functional Rehabilitation of a Patient with Abrasion, Attrition, and Missing Teeth
}

\author{
Rahul S Kulkarni
}

\begin{abstract}
The loss of tooth substance due to attrition and abrasion may lead to sensitivity to hot and cold, compromised esthetics, loss of anterior guidance and decreased masticatory efficiency. This case report describes the oral rehabilitation of a patient with abrasion, attrition and missing teeth. Treatment consisted of endodontic treatments of multiple teeth followed by their full coverage restorations, and fixed partial dentures for replacement of missing teeth. Esthetic and functional rehabilitation resulted in patient satisfaction, and no complications were noted related to the treatment after 1 year.
\end{abstract}

Keywords: Full mouth rehabilitation, Severely worn dentition, Attrition, Abrasion, Anterior guidance, Ceramometal crowns and bridges, Function and esthetics.

How to cite this article: Kulkarni RS. Esthetic and Functional Rehabilitation of a Patient with Abrasion, Attrition, and Missing Teeth. Int J Prosthodont Restor Dent 2014;4(2):52-55.

\section{Source of support: Nil}

Conflict of interest: None declared

\section{INTRODUCTION}

Pathological tooth wear can be categorized as abrasion, erosion, and attrition according to its cause. Attrition refers to accelerated wear of occlusal and incisal surfaces of teeth due to grinding, abrasion denotes wear due to abnormal mechanical process like vigorous tooth brushing, whereas erosion is loss of tooth substance due to chemical process. ${ }^{1,2}$ Besides of these definitions, the cause of noncarious tooth structure loss is often multifactorial, which occurs in combination in most clinical conditions. It is important to identify and eliminate and/or control the factors that contribute to wear during treatment of the worn dentition. The loss of tooth substance may lead to occlusal disharmony, pulpal pathology, esthetic disfigurement, and decreased masticatory efficiency. ${ }^{3}$ Management of worn dentition often requires multidisciplinary approach involving disciplines

\footnotetext{
Associate Professor

Department of Prosthodontics, Nair Hospital Dental College Mumbai, Maharashtra, India

Corresponding Author: Rahul S Kulkarni, Associate Professor, Department of Prosthodontics, Nair Hospital Dental College, Mumbai, Maharashtra, India, Phone: 02223082714 e-mail: drrahulprostho@yahoo.com
}

like prosthodontics, endodontics, periodontics and other disciplines. Comprehensive treatment plan in such patients often involves endodontic therapy, gingivoplasty, extractions, correction of the vertical dimension, and restoration of the lost tooth structure.

\section{CASE REPORT}

A 56-year-old male patient presented with a complaint of sensitivity to hot and cold, unesthetic appearance of teeth, missing teeth and progressive dental wear (Fig. 1). The patient had difficulty during incising and mastication, desired restoration of teeth for improved esthetics and function, and had noncontributory medical history. Intraoral examination revealed severe abrasion of all maxillary teeth except right lateral incisor and canine (Fig. 2). Mandibular teeth severely affected by abrasion were central incisor, canine and premolars on right side, and central and lateral incisors on left side (Fig. 3). Attrition was evident in upper and lower anterior teeth with open bite articulation resulting in loss of anterior guidance, while premolars and molars remained unaffected from attrition. Mandibular first molars on both sides were extracted due to dental caries 6 months ago, whereas maxillary second left molar was treated endodontically and given cast metal crown 2 years ago. Patient had received glass ionomer cement restorations on premolars and molar of right side for abrasion 3 months ago. There was no supraeruption of maxillary molars despite of loss of their antagonist, occlusal plane was unaffected, and centric relation position was

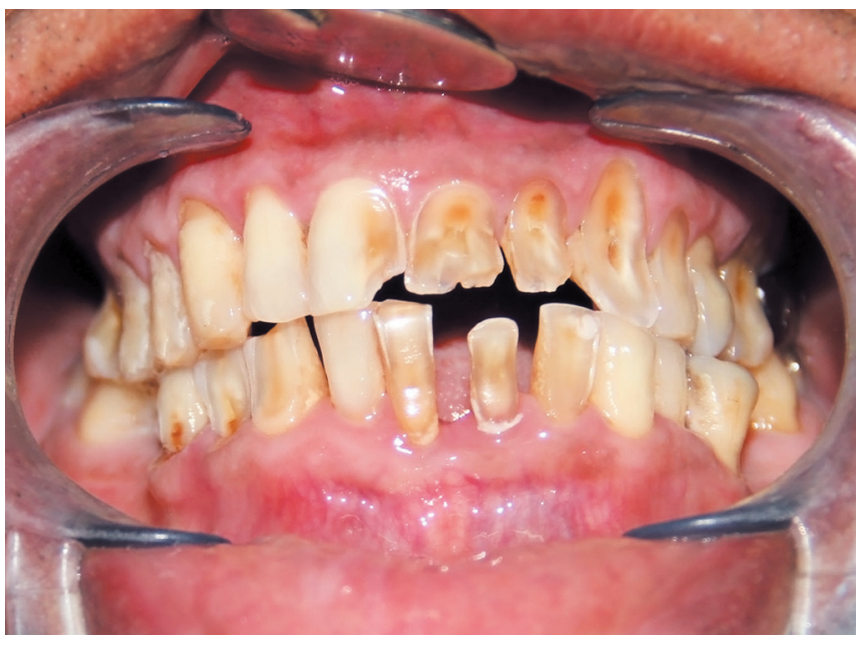

Fig. 1: Preoperative frontal view in centric occlusion 


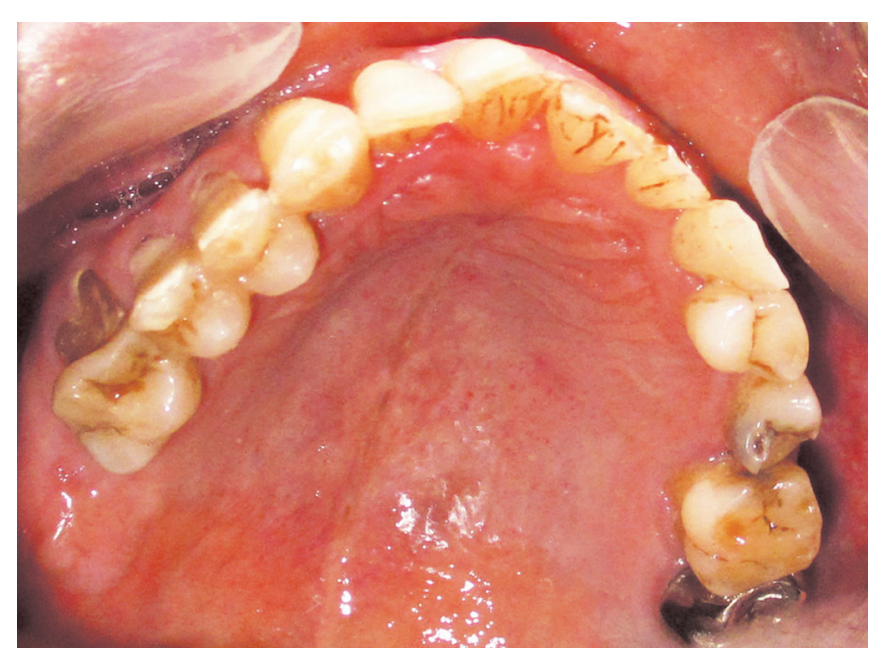

Fig. 2: Preoperative maxillary occlusal view

coincident with centric occlusion. Dietary history revealed that the patient was vegetarian, did not have high intake of citrus drinks and did not consume tobacco. Patient had habit of brushing teeth vigorously with hard bristle tooth brush twice a day which resulted in dental abrasion, and there was no history of bruxism. An important aspect of clinical examination was evaluation of the existing vertical dimension of occlusion (VDO), and assessment of need to increase the VDO in final restorations. ${ }^{4}$ This was carried out by evaluating the difference between vertical dimension of rest and occlusion, phonetic evaluation, patient's previous photographs and facial soft tissue contours. As previously mentioned, there was no attrition of premolars and molars, hence patient's original VDO was apparently maintained.

Diagnostic OPG revealed loss of enamel and dentin with periapical pathology of the abraded teeth, and adequate alveolar bone support to all the teeth including potential abutments for replacement of molars. The patient was given the following treatment options: root canal treatment and crowns for teeth severely affected by abrasion, and restoration of missing mandibular molars with dental implants or conventional fixed dental prostheses (FDPs). Patient declined the option of implants citing financial reasons, and consented for endodontic treatment and porcelain fused to metal (PFM) crowns on abraded teeth, and replacement of missing mandibular molars with FDPs.

Initial therapy consisted of oral prophylaxis, demonstration of scientific brushing technique, removal of etiologic factors (hard tooth brush and abrasive tooth paste), and dietary counseling. Teeth in which there was severe attrition and abrasion (e.g. maxillary left central and lateral incisors and canine) would present poor resistance and retention form after crown preparation. Hence, core build up was done in these, using core buildup material (LuxaCore, DMG, Hamberg, Germany). Diagnostic impressions were

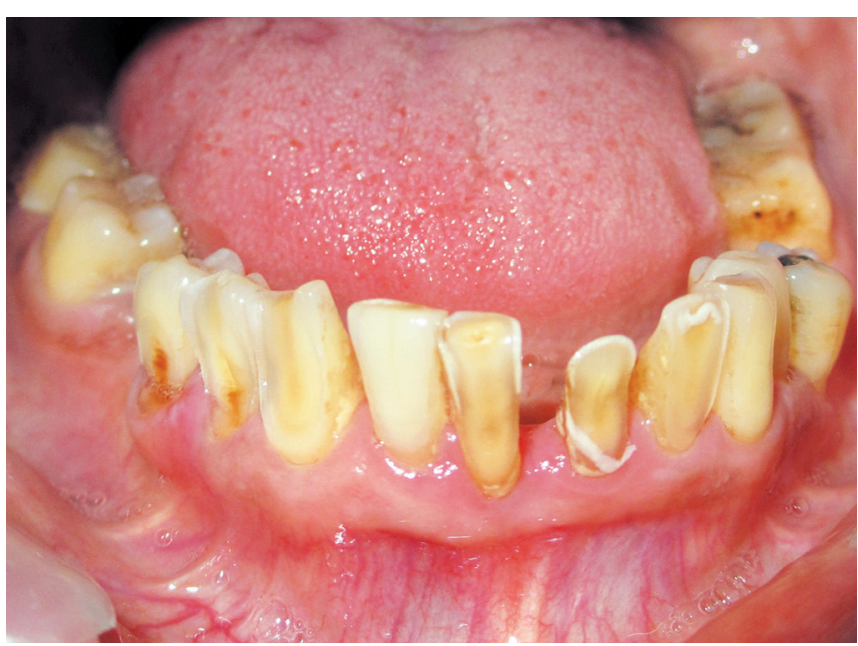

Fig. 3: Preoperative mandibular occlusofacial view; note missing mandibular first molars

made using alginate (Neocolloid, Zhermack, Badia Polesine, Italy) and poured in type III dental stone (Denstone, Kalabhai Karson, Mumbai, India). The patient's diagnostic casts were mounted on a semi-adjustable articulator (Hanau Wide-Vue, Whip Mix Corporation, Fort Collins, CO) using a face-bow record and an interocclusal record that was made with the aid of a Lucia jig and polyvinylsiloxane occlusal registration material (Exabite II; GC Corp., Tokyo, Japan). Protrusive records were obtained to set horizontal condylar inclination and Bennett angle. ${ }^{5}$ Mock preparation of maxillary and mandibular teeth was done on mounted diagnostic casts, and diagnostic wax-up was developed (Fig. 4). ${ }^{6}$ The anterior guidance and posterior disclusion on excursive movement were established in the diagnostic wax-up. ${ }^{7}$ Wax patterns were acrylized using tooth colored heat polymerized poly (methyl methacrylate) (PMMA) resin (Acrylin, DPI, Mumbai, India) following standard laboratory procedure, to form provisional restorations (Fig. 5). Maxillary and mandibular anterior and posterior teeth were prepared for metalceramic restorations using standard clinical protocol. ${ }^{8}$ Temporary restorations formed previously were lined with autopolymerizing PMMA resin (Alike Temporary C\&B Resin; GC America), and cemented with temporary luting cement (Freegenol, GC Corp., Tokyo, Japan). Centric occlusion, even protrusive contacts, canine guidance and disclusion of posterior teeth during eccentric movements of mandible were verified in the provisionals before discharging patient. The patient wore the provisional restorations for 3 months without complications. During the evaluation period, the patient's anterior and posterior speaking space and function were assessed. The muscles of mastication and the temporomandibular joint were evaluated for clinical signs of discomfort, and it was observed that the patient was asymptomatic and comfortable during this period. ${ }^{9}$ During the time of temporization, endodontic treatment was carried out 


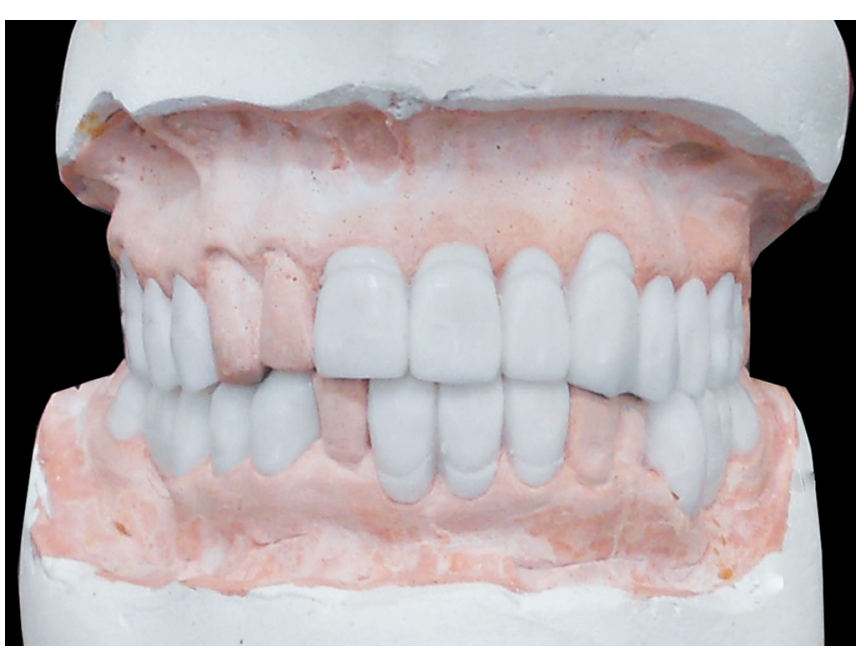

Fig. 4: Diagnostic wax-up

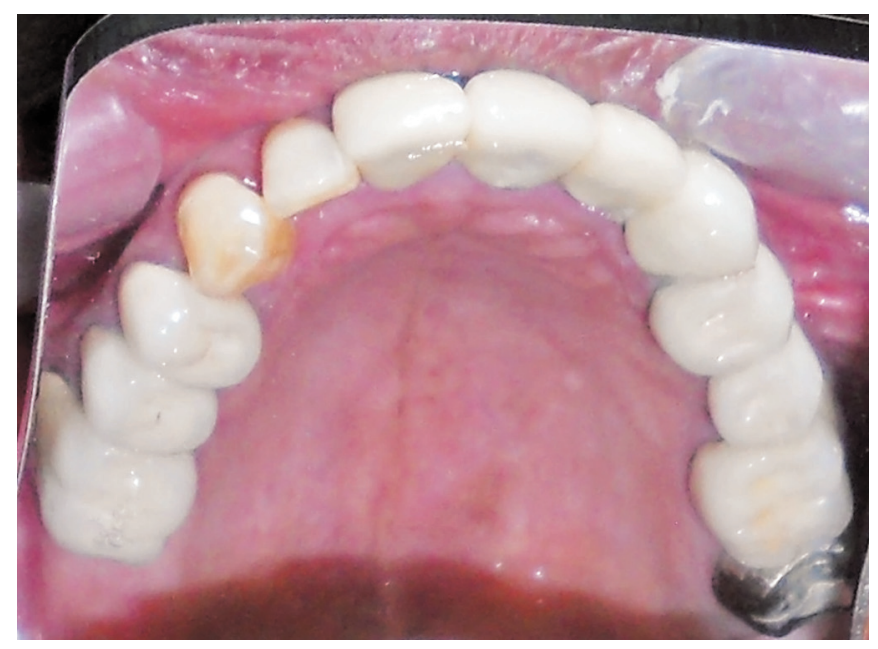

Fig. 6: Occlusal view of maxillary restorations

with the teeth pulpally involved by abrasion, using standard techniques. Mandibular right central incisor was found to be unsalvageable by endodontic treatment, hence, it was extracted and replaced by FDP. To record and preserve the anterior guidance of the provisional restorations, irreversible hydrocolloid impressions were obtained and poured in dental stone. Maxillary and mandibular casts were mounted to the semiadjustable articulator using face bow transfer and centric record. A custom incisal guide table was fabricated from acrylic resin (Pattern Resin LS; GC America) ${ }^{10}$ Final tooth modification and gingival retraction were carried out in the maxillary and mandibular arches, and definitive impressions were recorded using polyvinyl siloxane impression material (Affinis; Coltène/Whaledent Inc, Cuyahoga Falls, Ohio). Definitive casts were obtained using die stone. Bite registration was taken using provi-sional crowns and occlusal registration material (Stone Bite; Dreve Dentamid GmbH, Unna, Germany) in sections. Casts were transferred to the semiadjustable articulator using facebow and bite registrations. PFM restorations were made using customized anterior

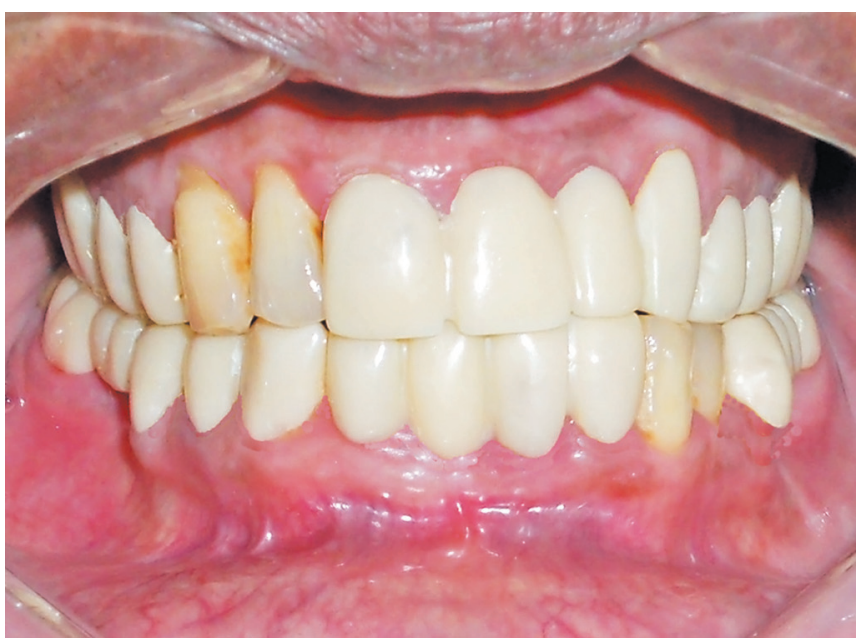

Fig. 5: Provisional restorations in place

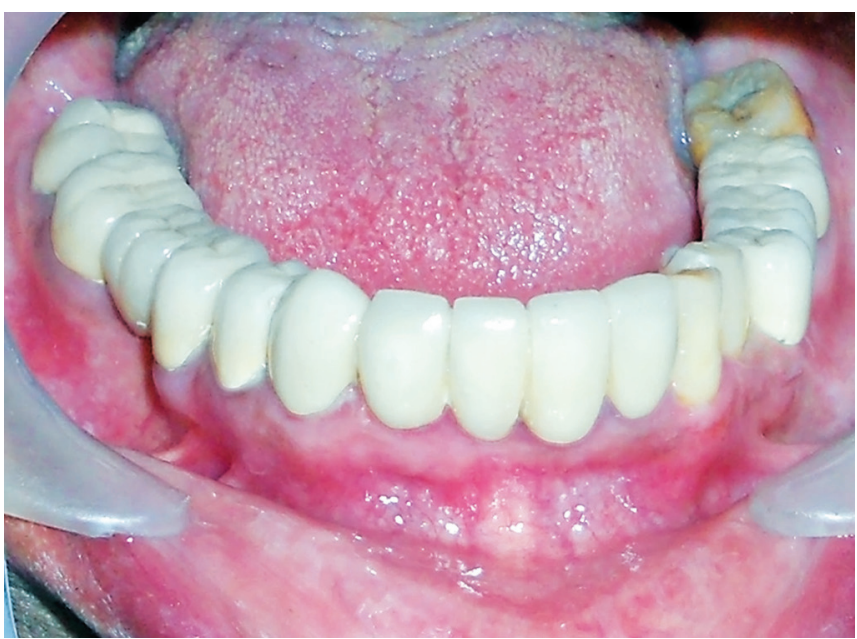

Fig. 7: Occlusofacial view of mandibular restorations

guide table fabricated previously. Individual crowns were made for abraded teeth, whereas 3 unit FDPs were made to replace mandibular molars on both sides. The prostheses were designed using mutually protected occlusion in which the anterior teeth protected the posterior teeth from excursive force and wear, and posterior teeth supported the bite force. The interocclusal space was ultimately evenly divided between the maxillary and mandibular arches at the time of definitive restorations. During bisque trial, centric occlusion, anterior guidance, and posterior disclusion were verified in the definitive restorations. Long centric occlusion was developed in the maxillary anterior restoration to allow for freedom in ante-rior posterior movement. This was followed by glazing of the ceramometal crowns, and finally their cementation with phosfate cement (Super Cement, Shofu, Kyoto, Japan) (Figs 6 to 8). Oral hygiene instructions were given and brushing technique was demonstrated. Recall evaluations were carried out at 3-month intervals for a period of 1 year. The patient's esthetic and functional expectations were satisfied, and he did not have sensitivity or pain after the treatment. 


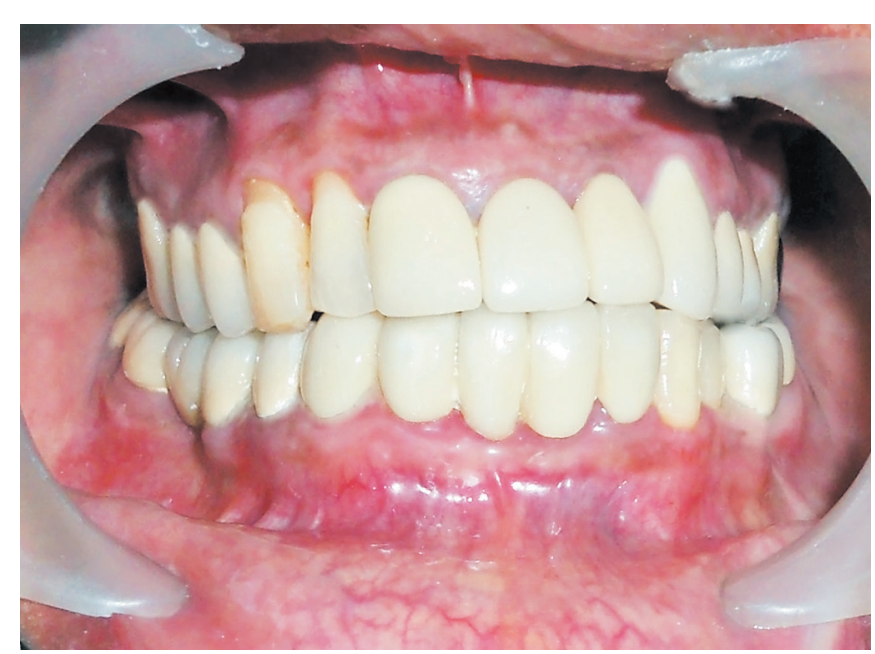

Fig. 8: Final restorations-centric occlusion

\section{CONCLUSION}

This clinical report describes the oral rehabilitation of a patient affected by abrasion, attrition and missing teeth. Endodontic treatment of pulpally involved teeth, and full coverage restorations resulted in alleviation of tooth sensitivity and improvement in esthetics. Restoration of the lost anterior guidance and replacement of missing teeth with PFM prostheses improved the masticatory efficiency.

\section{REFERENCES}

1. Smith BG. Toothwear: aetiology and diagnosis. Dent Update 1989;16:204-212.

2. The glossary of prosthodontic terms. J Prosthet Dent $2005 \mathrm{Jul}$; 94(1):10-92.

3. Turner KA, Missirlian DM. Restoration of the extremely worn dentition. J Prosthet Dent 1984 Oct;52(4):467-474.

4. Prasad S, Kuracina J, Monaco EA Jr. Altering occlusal vertical dimension provisionally with base metal onlays: a clinical report. J Prosthet Dent 2008 Nov;100(5):338-342.

5. Dawson PE. Simplifying instrumentation for occlusal analysis and treatment. In: Dawson PE, editor. Functional Occlusion From TMJ to smile design. 1st ed. St Louis: Mosby- Elsevier; 2007, p. 233-256.

6. Johansson A, Johansson AK, Omar R, Carlsson GE. Rehabilita-tion of the worn dentition. J Oral Rehabil 2008 Jul;35(7): 548-566.

7. Schuyler $\mathrm{CH}$. The function and importance of incisal guidance in oral rehabilitation 1963. J Prosthet Dent 2001 Sep;86(3): 219-232.

8. Rosensteil SF. Principles of tooth preparation. In: Rosensteil, Land, Fujimoto, editors. Contemporary fixed prosthodontics. 4 ed., St Louis: Mosby- Elsevier; 2006, p. 209-258.

9. Brown KE. Reconstruction considerations for severe dental attrition. J Prosthet Dent 1980 Oct;44(4):384-388.

10. Hoyle DE. Fabrication of a customized anterior guide table. J Prosthet Dent 1982 Oct;48(4):490-491. 